\title{
Silver Electrodeposited Carbon Nanofiber-Epoxy Electrode for Fluoxetine Detection
}

\author{
M. Ardelean, F. Manea, and R. Pode
}

\begin{abstract}
In this study, a simple and high sensitive electrochemical sensor is developed for the detection of fluoxetine (FXT) on a silver electrodeposited-carbon nanofiber-epoxy (AgCNFE) electrode. Cyclic voltammetry (CV) method was used for the comparative electrochemical behavior of FXT on AgCNFE, commercial silver electrode (Ag) and carbon nanofiber-epoxy (CNFE) composite electrode. A synergic effect in relation to the sensitivity for FXT detection was found on AgCNFE electrode versus commercial Ag electrode and CNFE electrode. Chronoamperometry (CA) results show that AgCNFE electrode exhibited a great potential for the real practical application in FXT detection without possible interference.
\end{abstract}

Index Terms-Carbon nanofiber, silver electrodeposited, fluoxetine detection.

\section{INTRODUCTION}

The utilization of nanostructured/nano-composite materials has been considered as a promising approach to design and develop new generation of chemical/biosensors, these materials being suitable for multiple applications [1]. Carbon based electrodes are widely used in electroanalytical investigations due to their chemical inertness, low background current and relative large potential window [2]. Among nanoscale carbonaceous materials, carbon nanotubes (CNTs) and carbon nanofibers (CNFs) have attracted the most research interests in the electroanalysis field. CNFs are considered to be a promising candidate to CNTs due to economic aspects and their similar electrocatalytic performance [1]-[3]. Moreover, CNF possesses less order and more edge sites on the outer wall that lead to more facile electron transfer. Also, better dispersion and wettability of $\mathrm{CNF}$ facilitates its manipulation to prepare CNF composite electrode [4]. CNT and CNF based composite electrodes have been reported for the detection of pentachlorophenol by our group [5], [6].

For various electroanalytical applications, it is required the presence of a catalyst that could be included in so-called chemically modified electrodes (CMEs). It is well-known that these electrodes possess electrocatalytic activity that is

Manuscript received May 22, 2015; revised July 12, 2015. This work was partially supported by the Romanian National Research Programs PN-II-Ideas-165/2011 and PNII-60/2012 and by the strategic grant POSDRU/159/1.5/S/137070 (2014) of the Ministry of National Education Protection, Romania, co-financed by the European Social Fund - Investing in People, within the Sectorial Operational Programme Human Resources Development 2007-2013.

M. Ardelean, F. Manea, and R. Pode are with Politehnica University of Timisoara, Romania (e-mail: magdalena.ardelean@upt.ro, florica.manea@upt.ro,rodica.pode@upt.ro). reflected as improved electroanalytical performance in the electroanalysis [7]-[9].

Silver inclusion within the electrode composition represents an useful and promising approach due to its high catalytic activity [7], [8]. The catalytic activity of the catalyst is in direct relation with its size and morphostructural characteristics. Smaller sizes and agglomeration avoiding lead to enhanced catalytic activity. There are several methods for Ag deposition on carbon nanotubes (CNTs), but the silver agglomeration mitigates their utility [10], [11].

Electrodeposition method is a very promising method to decorate nanostructured carbon dispersed within epoxy matrix with silver nanoparticles assuring their dispersion on the substrate [8].

The presence and the persistence of fluoxetine (FXT) in water makes as its detection to become very important from the point of view on the water pollution control. Besides common methods for FXT determination, the electroanalytical techniques using boron-doped diamond (BDD) electrode was previously reported [12]. Even if good sensitivity was achieved on BDD electrode, however the detection potential value of $+1.35 \mathrm{~V}$ vs. saturated calomel electrode (SCE) is too positive and other pharmaceuticals or organic pollutants from water could interfere it. Within this context, this study aims to characterize the electrochemical behavior by cyclic voltammetry of carbon nanofiber-epoxy (CNFE) and silver electrodeposited carbon nanofiber composite (AgCNFE) electrodes in comparison with silver commercial one in the presence of fluoxetine in water in order to detect it. The carbon nanofiber based composite electrodes were characterized morphologically and electrically. The electrochemical techniques for FXT detection were cyclic voltammetry (CV) and chronoamperometry (CA).

\section{EXPERIMENTAL DETAILS}

Carbon nanofiber-epoxy composite electrode (CNFE) consisted of $20 \%$ wt. CNF and $80 \%$ wt. epoxy resin with a surface area of $19.63 \mathrm{~mm}^{2}$ was obtained by two-roll mill method, which was detailed in our previous work [13]. Silver electrodeposition was performed by maintaining the composite electrode in $0.1 \mathrm{M} \mathrm{AgNO}_{3}$ solution at a potential value of $-0.4 \mathrm{~V} / \mathrm{SCE}$ for 120 seconds. Morphological characterization of the CNFs based composite electrodes was carried out using scanning electron microscopy (SEM XL20, Philips) with an acceleration voltage of $15 \mathrm{kV}$.

All the electrochemical studies were performed using an Autolab potentiostat-galvanostat PGSTAT 302 (Eco Chemie, The Netherlands) controlled by a PC using the GPES 4.9 software and a three-electrode cell at room temperature 
without further temperature control. The cell set-up included a working electrode, a platinum counter electrode and a saturated calomel reference electrode (SCE). Cyclic voltammetry $(\mathrm{CV})$ and chronoamperometry (CA) were used to assess the electrochemical performance of commercial $\mathrm{Ag}$ electrode, CNFE and AgCNFE, respectively. CNFE working electrode was mechanically polished with $0.2 \mu \mathrm{m} \mathrm{Al}_{2} \mathrm{O}_{3}$ powder, washed with distilled water, and then electrochemically pre-treated by ten repeated cycles. The supporting electrolyte was $0.1 \mathrm{M} \mathrm{Na}_{2} \mathrm{SO}_{4}$ solution, prepared from $\mathrm{Na}_{2} \mathrm{SO}_{4}$ of analytical purity (Merck, Germany) and distilled water.

The fluoxetine (Lilly, Pantheon, France) stock solution of 1 $\mathrm{mM}$ was prepared using ethanol (PAM Corporation, Romania) and $0.1 \mathrm{M} \mathrm{NaOH}$ (Merck, Germany) in a volume ratio of $1: 1$.

\section{RESULTS AND DISCUSSIONS}

In the Fig. 1 are presented the SEM images for the surface of AgCNFE electrode (Fig. 1(a)) in comparison with CNFE electrode (Fig. 1(b)). It can be easily noticed the uniform distribution of CNFs within the epoxy matrix for assuring a good electrical conductivity. Also, a good dispersion of silver particles onto carbon nanofibers was noticed. The value of electrical conductivity determined by four-point probe (FPP) method [14] was of $0.247 \mathrm{Scm}^{-1}$ for CNFE electrode that recommended it for electrochemical applications by type of the electrodeposition and the electroanalysis.

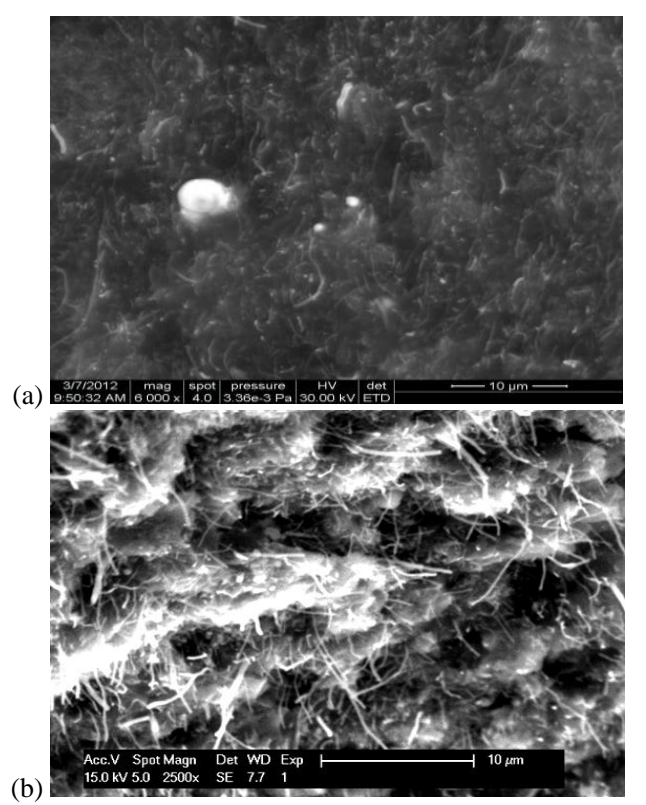

Fig. 1. (a) SEM image of AgCNFE electrode surface; (b) SEM image of CNFE electrode surface.

\section{A. Cyclic Voltammetry Measurements}

In order to obtain information about electrochemical behavior of the prepared electrodes, cyclic voltammetry technique was applied. For comparison, commercial Ag electrode was studied electrochemically using $\mathrm{CV}$ in the presence of FXT, the corresponding cyclic voltammograms being presented in Fig. 2(a). At a potential value of +0.3 V/SCE, a good linearity between anodic current and FXT concentration is achieved. Also, the cathodic peak appeared at about $+0.23 \mathrm{~V} / \mathrm{SCE}$ inform about the reversibility of FXT oxidation process.

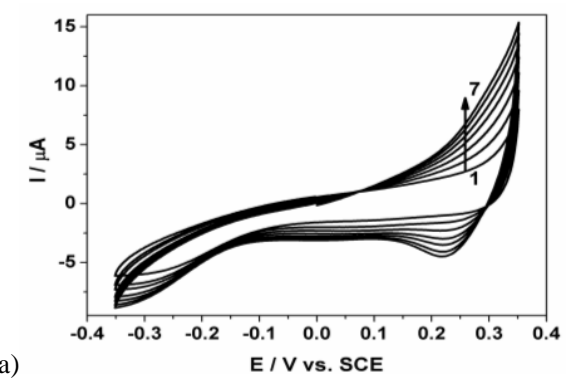

(a)

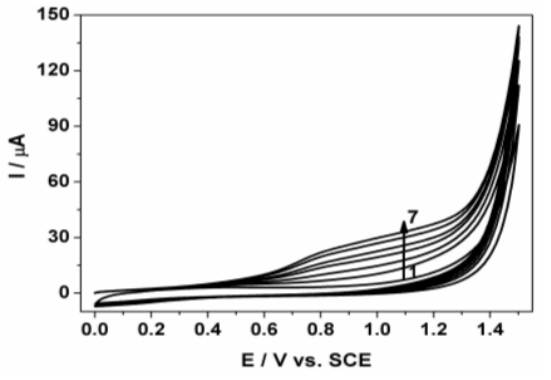

Fig. 2. (a) Cyclic voltammograms recorded at $\mathrm{Ag}$ electrode in $0.1 \mathrm{M} \mathrm{Na}_{2} \mathrm{SO}_{4}$ supporting electrolyte (curve 1) in the presence of 1-6 $\mu \mathrm{M}$ FXT (curves 2-7) at the potential scan rate of $0.05 \mathrm{Vs}^{-1}$ in a potential range from -0.35 to +0.35 V/SCE; (b) Cyclic voltammograms recorded at CNFE electrode in $0.1 \mathrm{M}$ $\mathrm{Na}_{2} \mathrm{SO}_{4}$ supporting electrolyte (curve 1 ) in the presence of 1-6 $\mu \mathrm{M}$ FXT (curves 2-7) at the potential scan rate of $0.05 \mathrm{Vs}^{-1}$ in a potential range from 0 to $+1.5 \mathrm{~V} / \mathrm{SCE}$.

For CNFE electrode, FXT oxidation process occurred at more positive potential value (about $+1 \mathrm{~V} / \mathrm{SCE}$ ), and no corresponding cathodic peak occurred, which should be explained by the irreversibility of FXT oxidation at this electrode. The anodic oxidation current increased linear with FXT concentration and a sensitivity of $3.868 \mu \mathrm{A}_{\mu} \mathrm{M}^{-1}$ was obtained, in comparison with $0.962 \mu \mathrm{A}_{\mu} \mathrm{M}^{-1}$ for commercial Ag electrode.

Nevertheless, the detection potential value of $+1 \mathrm{~V} / \mathrm{SCE}$ is still too high. It must be kept in mind that low detection potential value and good sensitivity are desired for the electroanalysis. To investigate the electrochemical behavior of AgCNFE electrode in the presence of various concentrations of FXT, the extended potential range corresponding to CNFE was selected for CV running and the results are presented in Fig. 3(a). It can be noticed that CV shape exhibited characteristics to the presence of silver and CNF by the anodic current peaks corresponding to FXT oxidation involving silver recorded at the potential value of $+0.3 \mathrm{~V} / \mathrm{SCE}$ and further oxidation of FXT direct on CNF that started at the potential value of about $+0.6 \mathrm{~V} / \mathrm{SCE}$. To compare the sensitivity for FXT detection with the results of commercial Ag electrode, the inset of Fig. 3(a) shows the CVs recorded for a narrow potential range. The calibration plots of the currents vs. FXT concentration and, implicit, the sensitivities are presented in Fig. 3(b). The results show a synergic effect for the sensitivities in relation with each single commercial Ag and CNFE composite electrode. Thus, the sensitivity of $5.816 \mu \mathrm{A} \mu \mathrm{M}^{-1}$ was obtained at the potential value of +0.324 V/SCE for AgCNFE electrode in comparison with $0.962 \mu \mathrm{A}_{\mu} \mathrm{M}^{-1}$ for commercial $\mathrm{Ag}$ electrode and 6.252 $\mu \mathrm{A} \mu \mathrm{M}^{-1}$ was obtained at the potential value of $+1.167 \mathrm{~V} / \mathrm{SCE}$ vs. $3.868 \mu \mathrm{A} \mu \mathrm{M}^{-1}$ for CNFE electrode. 


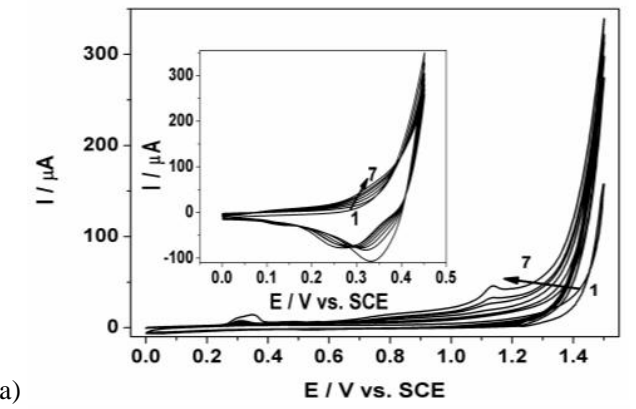

(a)

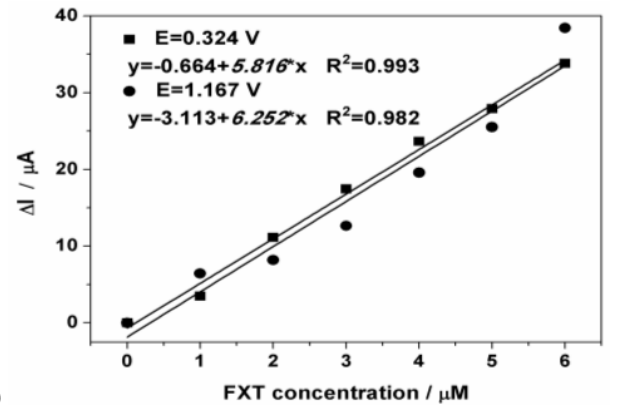

(b)

Fig. 3. (a). Cyclic voltammograms recorded at AgCNFE electrode in $0.1 \mathrm{M}$ $\mathrm{Na}_{2} \mathrm{SO}_{4}$ supporting electrolyte (curve 1) in the presence of 1- $6 \mu \mathrm{M}$ FXT (curves 2-7) at the potential scan rate of $0.05 \mathrm{Vs}^{-1}$ in a potential range from 0 to $+1.5 \mathrm{~V} / \mathrm{SCE}$; Inset: Cyclic voltammograms recorded at AgCNFE electrode in $0.1 \mathrm{M} \mathrm{Na}_{2} \mathrm{SO}_{4}$ supporting electrolyte (curve 1) in the presence of 1-6 $\mu \mathrm{M}$ FXT (curves 2-7) at the potential scan rate of $0.05 \mathrm{Vs}^{-1}$ in a potential range from 0 to $+0.45 \mathrm{~V} / \mathrm{SCE}$; (b). Calibration plots of the currents recorded at $+0.324 \mathrm{~V} / \mathrm{SCE}$ and $+1.167 \mathrm{~V} / \mathrm{SCE}$ vs. FXT concentration.

\section{B. Detection Measurements - Chronoamperometry}

Based on the voltammetric results, the easiest electrochemical technique, chronoamperometry was applied for two values of detection potential of $+0.35 \mathrm{~V} / \mathrm{SCE}$ and +1.1 $\mathrm{V} / \mathrm{SCE}$ in the presence of various FXT concentrations. The results are presented in Fig. 4(a). Because the current response was lower at the potential value of $+0.35 \mathrm{~V} / \mathrm{SCE}$, a detail of the chronoamperograms is presented in the inset of Fig. 4(a). A good linearity of current- FXT concentration was observed for both potential values, with good correlation coefficients.

(a)
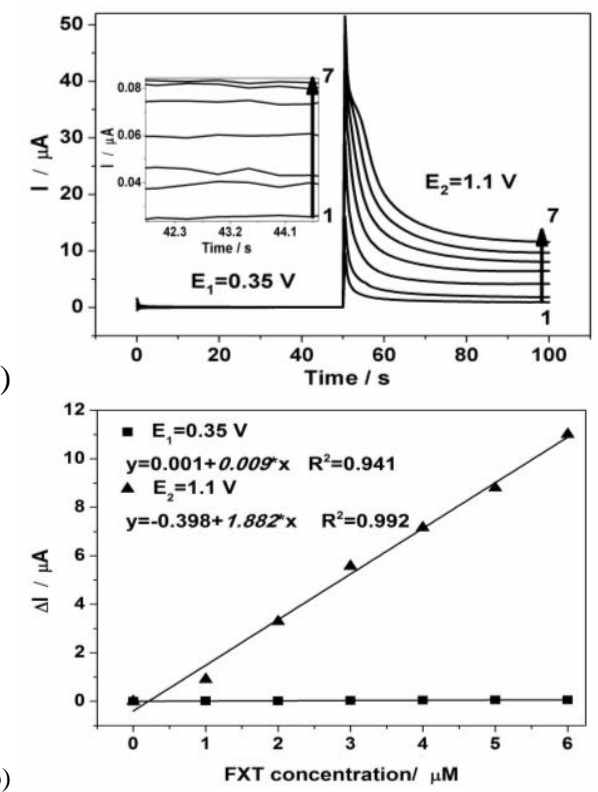

(b)

Fig. 4. (a) Chronoamperograms recorded at the potential values of $\mathrm{E}_{1}=+0.35 \mathrm{~V} / \mathrm{SCE}$ and $\mathrm{E}_{2}=+1.1 \mathrm{~V} / \mathrm{SCE}$ at $\mathrm{AgCNFE}$ electrode in $0.1 \mathrm{M}$ $\mathrm{Na}_{2} \mathrm{SO}_{4}$ supporting electrolyte (curve 1) in the presence of 1-6 $\mu \mathrm{M}$ FXT (curves 2-7); Inset: Detail of chronoamperograms recorded at the potential value of $+0.35 \mathrm{~V} / \mathrm{SCE}$; (b) Calibration plots of the currents vs. FXT concentration.
The electroanalytical parameters obtained by employing the voltammetric and amperometric techniques are hathered in Table I.

TABLE I: THE ELECTROANALYTICAL PARAMETERS FOR FXT DETECTION DETECTION USING AGCNFE ELECTRODE

\begin{tabular}{lcccccc}
\hline \hline Tech. & $\begin{array}{c}\mathrm{E}_{\text {det }} \\
(\mathrm{V} / \mathrm{SCE})\end{array}$ & $\begin{array}{c}\text { Sens. } \\
(\mu \mathrm{A} / \mu \mathrm{M})\end{array}$ & $\mathrm{R}^{2}$ & $\begin{array}{c}\text { RSD } \\
(\%)\end{array}$ & $\begin{array}{c}\text { LOD } \\
(\mu \mathrm{M})\end{array}$ & $\begin{array}{c}\text { LOD } \\
(\mu \mathrm{M})\end{array}$ \\
\hline $\mathrm{CV}$ & 0.324 & $\mathbf{5 . 8 1 6}$ & 0.993 & 0.73 & 0.081 & 0.270 \\
& 1.167 & $\mathbf{6 . 2 6 2}$ & 0.982 & 4.82 & 0.189 & 0.631 \\
\hline $\mathrm{CA}$ & 0.350 & 0.009 & 0.992 & 24.7 & 2.380 & 7.934 \\
& 1.100 & 1.882 & 0.992 & 5.39 & $\mathbf{0 . 0 7 9}$ & $\mathbf{0 . 2 6 6}$ \\
\hline \hline
\end{tabular}

It can be noticed that the best sensitivity was reached using $\mathrm{CV}$, which allowed to detect FXT at low potential value $(+0.324 \mathrm{~V} / \mathrm{SCE})$ characterized by similar sensitivity to the one obtained at higher potential value $(+1.167 \mathrm{~V} / \mathrm{SCE})$. This aspect is very important because mitigates the interference potential. CA operated at low potential value is not satisfactory due to very low sensitivity that can be explained by the catalyst fouling. However, the lowest limit of detection was achived by employing CA at $+1.1 \mathrm{~V} / \mathrm{SCE}$ which is very promising for very low concentrations of FXT.

\section{CONCLUSIONS}

The silver electrodeposited-carbon nanofiber-epoxy (AgCNFE) composite electrode obtained by silver electrodeposition on CNFE composite electrode exhibited the synergic effect in relation to the sensitivity for fluoxetine detection in aqueous solution in comparison with commercial silver electrode (Ag) and carbon nanofiber-epoxy (CNFE) composite electrode.

Both voltammetric and amperometric techniques applied and certain operating conditions allowed good performances for FXT detection at AgCNFE.

The results of this study are very promising for the practical application for fluoxetine detection in the aqueous solution. The selection of voltammetric or amperometric technique and the operating detection potential will be related to the concrete practical applications.

\section{REFERENCES}

[1] Z. Lin, L. Ji, and X. Zhang, "Electrocatalytic properties of Pt/carbon composite nanofibers," Electrochim Acta, vol. 54, no. 27, pp. 7042-7047, 2009.

[2] M. Zhou, J. Guo, L. Guo, and J. Bai, "Electrochemical sensing platform based on the highly ordered mesoporous carbon-fullerene system," Anal. Chem., vol. 80, no. 12, pp. 4642-4650, 2008.

[3] Y. Liu, D. Wang, J. Huang, H. Hou, and T. You, "Highly sensitive composite electrode based on electrospun carbon nanofibers and ionic liquid," Electrochem. Commun., vol. 12, no. 8, pp. 1108-1111, 2010.

[4] Wu L, Zhang X, and Ju H, "Detection of NADH and ethanol based on catalytic activity of soluble carbon nanofiber with low overpotential," Anal Chem, vol. 79, no. 2, pp. 453-458, 2007.

[5] A. Baciu, F. Manea, A. Remes, S. Motoc, G. Burtica, and R. Pode, "Anodic determination of pentachlorphenol from water using carbon nanofiber-based composite electrode," Environ. Eng. Manag. J., vol. 9, no. 11, pp. 1555-1562, 2010.

[6] A. Remes, A. Pop, F. Manea, A. Baciu, S. J. Picken, and J. Schoonman, "Electrochemical determination of pentachlorphenol in water on multi-wall carbon nanotubes-epoxy composite electrode," Sensors, vol. 12, no. 6, pp. 7033-7046, 2010.

[7] B. Habibi and M. Jahanbakhshi, "Silver nanoparticles/multi walled carbon nanotubes nanocomposite modified electrodes: Voltammetric 
determination of clonazepam," Electrochim. Acta, vol. 118, pp. 10-17, 2014.

[8] F. Manea, S. Motoc, A. Pop, A. Remes, and J. Schoonman, "Silver-functionalized carbon nanofiber composite electrodes for ibuprofen detection," Nanoscale Res. Lett., vol. 7, pp. 331-335, 2012.

[9] Y. Liu, D. Wang, Y. X, H. Hou, and T. You, "A novel and simple route to prepare Pt nanoparticle-loaded carbon nanofiber electrode for hydrogen peroxide sensing," Biosens. Bioelectron., vol. 26, no. 11, pp. 4585-4590, 2011.

[10] F. Xin and L. Li, "Decoration of carbon nanotubes with silver nanoparticles for advanced CNT/polymer nanocomposites," Composites: Part A, vol. 42, pp. 961-967, 2011.

[11] Y. Liu, J. Tang, W. Chen, G. K. H. Pang, and J. K. Xin, "A wet-chemical route for the decoration of CNTs with silver nanoparticles," Carbon, vol. 44, no. 2, pp. 381-392, 2006.

[12] M. Ardelean, F. Manea, and R. Pode, "Electrochemical detection of fluoxetine using a boron-doped diamond electrode," Int. J. Pharm. Pharm. Sci., vol. 5, no. 3, pp. 318-322, 2013.

[13] M. Ardelean, F. Manea, N. Vaszilcsin, and R. Pode, "Electrochemical detection of sulphide in water/seawater using nanaostructured carbon-epoxy composite electrodes," Anal. Meth., vol. 6, pp. 4775-4782, 2014.

[14] V. S. Mironov, J. K. Kim, M. Park, S. Lim, and W. K. Cho, "Comparison of electrical conductivity data obtained by four-electrode and four-point probe methods for graphite-based polymer composites," Polym Test., vol. 26, no. 4, pp. 547-555, 2007.

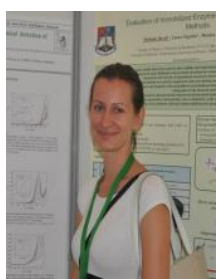

M. Ardelean completed her $\mathrm{PhD}$ in chemical engineering at Politehnica University of Timisoara, Romania in 2015.

During her PhD stage, she was actively involved in a Swiss-Romanian Joint Research Program - Impact of waste and pollutants on environment and climate: Collaborative research study of the wastewater discharge impact in the Bega-Timiș River Sub-Basins.
Her research interests are focused on water quality, e.g., water monitoring, micropollutants -pharmaceutical compounds. She has published 3 research articles and presented her research results at several international conferences.

F. Manea was employed in 1999 and currently she activates as a professor of environmental engineering at the Department of Applied Chemistry and Engineering of Inorganic Compounds and Environment, Faculty of Industrial Chemistry and Environmental Engineering, Politehnica University of Timisosra, Romania. Her research interest is focused on advanced electrode materials and electrochemical techniques applied in electroanalysis/sensors.

R. Pode is a professor at University Politehnica Timisoara, Faculty of Industrial Chemistry and Environmental Engineering and a $\mathrm{PhD}$ coordinator in the following domains: new catalysts applied in heterogeneous photocatalytic processes; advanced oxidation processes in relation with water treatment and quality control. Her principal subjects/occupational skills covered are engineering of wastewater treatment processes; advanced oxidation processes; new catalysts applied in heterogeneous photocatalytic processes; physico-chemical methods for municipal and industrial sludge treatment. 